\title{
Permissiveness Paradox in Cell Cycle Progression Towards Genome Instability and Malignant Transformation
}

\author{
Lawrence M Agius* \\ Department Of Pathology, Mater Dei Hospital, Tal-Qroqq, University Of Malta Medical School, Msida, Malta Europe
}

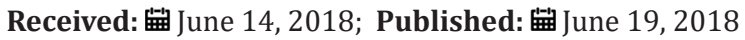

*Corresponding author: Lawrence M Agius, Department Of Pathology, Mater Dei Hospital, Tal-Qroqq, University Of Malta Medical School, Msida, Malta Europe

\begin{abstract}
Dimensional representation of events actively executing the cell cycle dynamics of progression in carcinogenesis allow for a paradoxical emergence of highly permissive elements in the establishment of the mitotic spindle and the separation of chromatids. In such terms, ongoing processes of dissolution of the nuclear membrane betray an essential permissiveness in cell cycling that is paramount constitutional characterization of a process as "passenger" event in defining malignant transformation. In real terms, incremental progression of the cell cycle is specific hallmark for the events that constitutionally permit the system machinery in mitosis to conclusively establish potential re-setting of events as non-re-replication of the cell cycle in the re-emerging G1 phase of mitotic activity.
\end{abstract}

\section{Introduction}

The cell cycle dynamics and its relative interactions with states of instability of the genome acquire significant relevance in regard to cellular malignant transformation. There appears to be a tumor suppressor role effect of apoptosis, DNA repair by homozygous recombination and cell cycle arrest in G2 in simple epithelia subject to ionizing radiation-induced DNA damage [1]. The attributes of such interface effects include the dimensional shifts in phosphorylation of the retinoblastoma suppressor gene activities in a manner that prevents re-replication of the nuclear DNA within the same cell cycle. Such processes are linked to inhibitory effects of phosphorylation in relative measure to rephosphorylation. The dimensional connotations relative to genomic instability underlie progression of a cell-cycle series of events relative to the stabilization of CDK1/Cyclin B1 that progresses as alternate modes of representation and constitution of the cell replicative machinery components. In such measure, the inclusion of integral genomic participation in each of the cell cycle stages allows for phosphorylation and other post-translational events to assume transforming attributes to stage identity and potential. The bacteriome in mammals may produce genotoxins, express pathways with the production of free radicals or affect DNA repair causing genome damage, cell cycle arrest and apoptosis, modulate immunity and provoke carcinogenesis in the host organism [2]. Lineage tracing may contribute to dissecting the embryonic origin of brain neoplasms and lineage-specific mechanisms regulate stem cell behavior that may be subverted in tumorogenesis [3]. In real terms, the inclusion of substantial dimensional dynamics includes in particular the dissolution of the nuclear lamina and membrane into the cytoplasm. It is further to recognition of nuclear targeting that the $\mathrm{Rb}$ suppressor pathway assumes significance in the establishment of malignant transformation. Biallelic ablation of the $\mathrm{Rb}$ gene pathway is tantamount confirmation of a cell-cycle dynamics that corresponds to the emergence of DNA lesions as responding stimulus to both replicative and trans-lesion polymerase activity.

\section{Kinetics}

Metabolic reprogramming is pivotal to sustain cancer growth and progression, and autophagy is a major pathway controlling cell metabolism [4]. The kinetics of the mitotic phase in cell division equate to the progression of the G1 phase asa well-studied process of progression of cell proliferative potential. The actin-binding 
protein MISP controls spindle orientation and mitotic progression in human cells [5]. The inclusion of cyclin/CDK produces the "passenger" dynamics of a process of incremental transformation as well-illustrated also by the short duration of the G2 phase of the cell cycle. In likemanner, the dimensions of inclusion of mitotic phase progression are integral to the potential for genomic instability as indeed portrayed by the trans-lesion polymerases that show less fidelity measures than the replicative polymerases. Appropriate setting and re-setting of the mitotic cell cycle include the progression of the cell mitotic spindle as indeed projected in terms of systems of adaptor proteins and as coordinating co-activators and co-repressors. The dynamic behavior of microtubules constitutes the basis of cellular motility, cytoplasmic transport and cell mitosis [6]. Indeed proteolysis is central to a series of corresponding post-translational modifications as well exemplified by dynamics and activation/suppressor states of the $\mathrm{Rb}$ suppressor protein. It is further to such dimensional increment in progression of the cell cycle that the transforming potential for further progression in transformation proves instrumental and persistent in terms of genomic instability. Altered tubulin isotype of microtubules is emerging as a feature of aggressive and treatment refractory cancers [7].

\section{Proportional Representation}

Proportional representation of components of the cell cycle machinery allows for the establishment of subunit participation in incremental progression of DNA synthesis and transition to duplication of the nuclear DNA. The PC/C-Cdh2 ubiquitin-ligase complex targets cell cycle regulators for proteosomal degradation and helps prevent tumorogenesis and the accumulation of chromosomal aberrations [8]. Synthesis progression permits the realization for further increments in activity of cyclins/CDKs within the context for further establishment of the cell-cycle spindle and of the metaphase plate. Induction of events is best portrayed by the introduction of events in the elaboration of injury to the genome in terms that implicate genome instability.

The dynamics of the mitotic phase is relative to the processes of DNA synthesis in the $\mathrm{S}$ phase and is representative constitutional identity of the whole phenomenon of creation of two daughter cells. It is feasible to distinguish tumors driven by strong positive sub-clonal selection from those evolving neutrally or under weak selection, as the latter fail to dramatically alter sub-clonal composition [9]. The representative projection pathways include the DNA condensation and the separation of chromatids. Perceptual identity promotes the further incremental recruitment of agents as factors specific to the emergence of anaphase and metaphase stages of mitotic separation of the chromatids from the metaphase plate. In such manner, the distributional realization of two daughter cells is confirmatory dimension of the synthetic phase of the cell cycle. It is possible that one or more cellular kinetic parameters of hematopoietic stem cells (HSCs) are affected by inflammation, such as cell division or death rates of cells and the probability of HSC differentiation [10].

\section{Persistence of Progression}

Persistence of the incremental progression of a given cell cycle confirms the pathogenic prevention of re-replication as well illustrated by the emergence of phosphorylation states that conclusively inhibit translational modifications on the one hand and proteolysis as further projected by the later phases of the cell cycle. During the gap phases G1 and G2, mitochondria form an interconnected network whereas in mitosis and S-phase fragmentation of the mitochondrial network will take place [11]. Mitochondrial dynamics has been implicated in malignant properties of cells, particularly metastasis [12]. In such measure, the distributional kinetics of injury to the DNA allow for permissive re-setting of cell cycle dynamics as confirmed by the Rb suppressive effects on gene expression as induced by E2F dynamics. A mechanism may exist whereby transcription-coupled eviction of cohesion from CDKN1A might act as a molecular switch to control spatial interactions between regulatory genomic elements [13].

\section{Paradox of Permissiveness}

It is paradoxical that suppressive and non-suppressive permissiveness of various factors pathogenic of genomic instability states alternatively allows for the emergence of confronting dimensions of post-translational events relative to proteolysis in later stages of cell cycle progression. The realization of systems of permissive re-combination of component factors is directional consequence to the emergence of DNA injury in terms of the dynamics of the cell cycle itself. Septins are GTP-binding cytoskeletal proteins that contribute to a broad range of tumor types and pharmaceutical inhibition of global septin dynamics greatly suppresses proliferation, migration and invasiveness in breast cancer cell lines [14]. It is towards further progression of mitosis that the system pathways of increment in genome instability prove effective in establishment of the cell cycle in the effective phase of suppressed re-replication of the mitotic process.

\section{Concluding Remarks}

Revealing elements of induction pathways in the essential progressiveness of injury of the DNA in further evolution of the individual cell cycle is tantamount evidence for the emergence of such DNA damage in terms that implicate permissive phenomena. Post-translational modifications alternate and also integrate with the essential pathogenesis of tumorogenesis as a permissive process of ongoing proteolysis. It is further to such increments that two daughter cells that are replicas of the original parent cell that involves inclusion dynamics of mitotic spindle formation and separation of the chromatids produced. The further confirmatory dimensions of such dual sets of process events allow for the emergence of permissive potential towards carcinogenesis. MIIP, a cytoskeleton regulator, blocks cell migratory invasiveness and delays mitosis with suppression of tumorigenesis [15]. Distributional dynamics of CDKs/Cyclins conform to a specific re-setting of events that potentially re-organize the component pathways within gap phases of progression as well projected by the 
systems of dissolution of the nuclear membrane and as systems of nuclear dissolution in further loss of delineation of the nucleus from the cytoplasm. It is evidentially apparent that the essential potential for non-re-replication is fundamental to stages of re-setting in the face of further progression of the cell cycle itself. Clinical trials investigating the impact of checkpoint inhibitors in Epstein-Barr virus associated gastric cancer are currently underway [16]. Significant attainment of telomerase or alternative lengthening of telomeres may maintain telomere length and may be permissive and required for clonal evolution of genomically-unstable cells during progression to malignancy [17].

The prevention of re-replication during the essential progression of a given cell cycle event corresponds to the emergence of integral dimensions of genome integrity or alternatively of genomic instability per se. Proportional representation of individual components of the cell cycle machinery allows for the essential proteolysis of such components as derivative hallmarks of an injury to the DNA within such systems as nuclear dissolution and chromatid separation during the mitosis phase of the cell cycle. Intrinsically disordered and misfolded proteins and the process of carcinogenesis involving viral pathogens are closely related, including such pathogens as human papilloma virus [18]. In terms beyond simple re-constitution of events in cell division, it is the performance of stimulus participation that incremental progression is only one facet in the induction phenomenon as exerted by DNA damage in facilitating progression to the mitosis phase constitution of the cell cycle.

\section{References}

1. Dekanty A, Barrio L, Milan M (2015) Contributions of DNA repair, cell cycle checkpoints and cell death to suppressing the DNA damageinduced tumorigenic behaviour of Drosophila epithelial cells. Oncogene 34(8): 978-85.

2. Druzhinin VG, Matskova LV, Fucic A (2018) Induction and modulation of genotoxicity by the bacteriome in mammals. Mutat Res 776: 70-77.

3. Azzarelli R, Simons BD, Philpott A (2018) The developmental origin of brain tumors: a cellular and molecular framework. Development 145(10).

4. Wang L, Shang Z, Zhou Y, Hu X, Chen Y, et al. (2018) Autophagy mediates glucose starvation-induced glioblastoma cell quiescence and chemoresistance through coordinating cell metabolism, cell cycle, and survival. Cell Death Dis 9(2): 213.

5. Vodicska B, Cerikan B, Schiebel E, Hoffmann I (2018) MISP regulates the IQGAP1/Cdc42 couple to collectively orchestrate spindle orientation and mitotic progression. Sci Rep 8(1): 6330.

6. Cirillo L, Gotta M, Meraldi P (2017) The elephant in the room: the role of microtubules in cancer. Adv Ep Med Biol 1002: 93-124.

7. Parker AL, Teo WS, McCarroll JA, Kavallaris M (2017) An emerging role for tubulin isotypes in modulating cancer biology and chemotherapy Resistance. Int J Mol Sci 18(7).

8. Garson J, Rodriguez R, Kong Z, Chabes A, Rodriguez-Acebes S, et al (2017) Shortage of dNTPs underlies altered replication dynamics and DNA breakage in the absence of the PC/C cofactor Cdh1. Oncogene 36(42): 5808-5818.

9. Sun R, HuZ, Sottoriva A, Graham TA, Harpak A, et al. (2017) Betweenregion genetic divergence reflects the mode and tempo of tumor evolution. Nat Genet 49(7): 1015-1024.

10. Zhang J, Fleischman AG, Wodraz D, Komarova NL (2017) Determining the role of inflammation in the selection of JAK2 mutant cells in myeloproliferative neoplasms. J Theor Biol 425: 43-52.

11. Torbay R, Bilyy R (2016) Mitochondrial dynamics during cell cycling. Apoptosis 21(12): 1327-1335.

12. Fu L, Dong Q He J, Wang X, Xing J, et al. (2017) SIRT4 inhibits malignancy progression of NSCLCs, through mitochondrial dynamics mediated by the ERK-Drp1 pathway. Oncogene 36(19): 2724-2736.

13. Millau JF, Wijchers P, Gaudreau L (2016) High resolution 4C reveals rapid p53-dependent chromatin reorganisation of the CDKN1A locus in response to stress. PLoS One 11(10): e0163885.

14. Zhang N, Liu L, Fan N, Zhang Q, Wang W, et al. (2016) The requirement of SEPT2 and SEPT7 for migration and invasion in human breast cancer via MEK/ERK activation. Oncotarget 7(38): 61587-61600.

15. Wang Y, Wen J, Zhang W (2011) MIIP, a cytoskeleton regulator that blocks cell migration and invasion, delays mitosis, and suppresses tumorogenesis. Curr Protein Pept Sci 12(1): 68-73.

16. Naseem M, Barzi A, Brezden Masley C, Puccini A, Berger MD, et al. (2018) Outlooks on Epstein-Barr virus associated gastric cancer. Cancer Treat Rev 66: 15-22.

17. Bernal A, Tusell L (2018) Telomeres: implications for cancer development. Int J Mol Sci 19(1)

18. Tamarozzi ER, Giuliatti S (2018) Understanding the role of intrinsic disorder of viral proteinsin the oncogenicity of different types of HPV. Int J Mol Sci 19(1): 198.
This work is licensed under Creative Commons Attribution 4.0 License

To Submit Your Article Click Here: Submit Article
DOI: $10.32474 /$ OAJOM.2018.02.000133

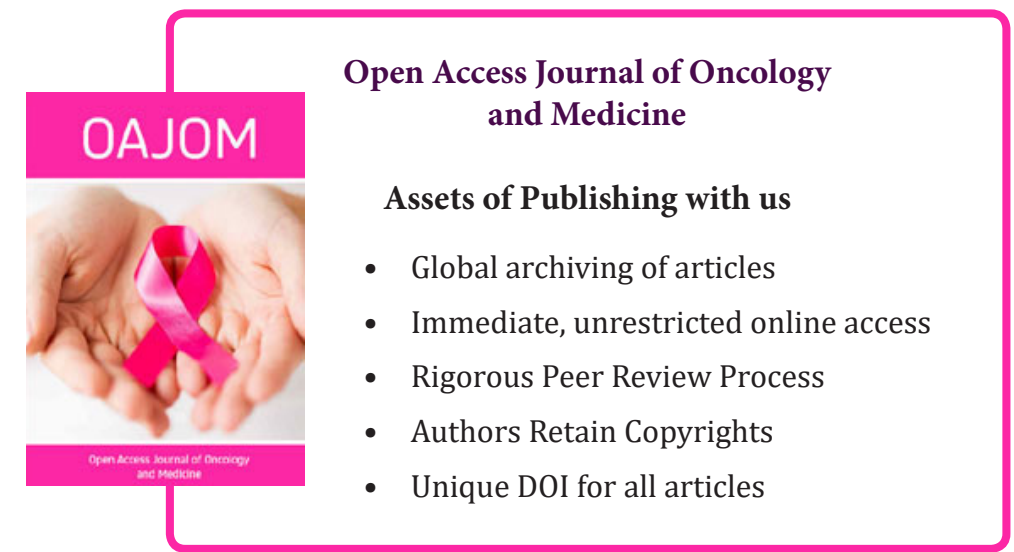

